\title{
Effect of ZnO Layers on Transport and Relaxation of Charge in Porous Silicon-Silicon Structures
}

\author{
I. B. Olenych \\ Department of Electronics, Ivan Franko Lviv National University, Ukraine
}

Copyright $\bigcirc 2016$ by authors, all rights reserved. Authors agree that this article remains permanently open access under the terms of the Creative Commons Attribution License 4.0 International License

\begin{abstract}
Zinc oxide nanostructures have been grown by electrochemical deposition on porous silicon-silicon substrate. The effect of electrolyte temperature on the morphology of grown $\mathrm{ZnO}$ arrays was observed. Temperature dependencies of the electrical conductivity for the structures based on porous silicon were investigated in 80-325 $\mathrm{K}$ range. The results are analyzed within the model of disordered semiconductors and the activation energy of charge transport is determined. It is shown that $\mathrm{ZnO}$ layers cause the decrease of the electrical conductivity activation energy in 140-250 $\mathrm{K}$ temperature range. Electric conductivity was also shown to be dependent on the morphology of $\mathrm{ZnO}$ arrays. Based on the spectra of thermally stimulated depolarization current, the localized electron states in the experimental samples are found. The trap levels are distributed quasi-continuously on the activation energy and exist in the ranges of $0.2-0.3,0.4-0.45$, $0.5-0.55$ and $0.6-0.65 \mathrm{eV}$. $\mathrm{ZnO}$ nanocrystals grown on porous silicon substrates modify density of states in different energy ranges.
\end{abstract}

Keywords Porous Silicon, Zinc Oxide, Conductivity, Activation Energy, Thermally Stimulated Depolarization

\section{Introduction}

With the rapid growth of nanotechnology, zinc oxide $(\mathrm{ZnO})$ gained significant importance as a functional semiconducting material. $\mathrm{ZnO}$ with its wide direct bandgap of $3.37 \mathrm{eV}$ and large exciton binding energy of $60 \mathrm{meV}$ is a prospective material for use in various electronic and optoelectronic applications [1-3]. As far as optoelectronics is considered, $\mathrm{ZnO}$ main benefits are green and blue luminescence, transparency of nanostructured $\mathrm{ZnO}$ layers in the visible, efficient photoelectric conversion in the UV, antireflective properties and compatibility with traditional electrode materials [4-8]. $\mathrm{ZnO}$ also shows interesting catalytic [9], sensor $[10,11]$ and piezoelectric properties $[3,12]$.
By exploiting the peculiarities of crystal structure one can effectively control the morphology of $\mathrm{ZnO}$ nanostructures in the fabrication process [13]. Diverse morphologies of $\mathrm{ZnO}$ nanostructures such as nanorods, nanowires, nanosheets, nanocombs and nanoribbons have been synthesized by different physical and chemical fabrication techniques. These fabrication methods include chemical vapor deposition [14], thermal evaporation [15,16], magnetron sputtering [17], hydrothermal [18], pulsed laser deposition $[19,20]$, sol-gel [21,22], chemical bats deposition [23], flame transport synthesis [24] and electrochemical deposition $[5,8,25]$. Many reports have thoroughly discussed the growth mechanism and explained the differences in the structures obtained via different technological routes.

Porous silicon (PS) is a perfect candidate to serve as a substrate for the deposition of $\mathrm{ZnO}$ nanocrystals, since sponge-like geometrical structure of PS allows for $\mathrm{ZnO}$ to infiltrate into the substrate with large specific surface area. It is generally thought that PS provides an advantage for growing high-quality $\mathrm{ZnO}$ nanostructured arrays, decreasing mechanical stress caused by large mismatches in the lattice constant and thermal expansion coefficients [26].

PS is prepared by etching a single Si crystal with the formation of small cavities, resulting in thick walls between the pores that may have several nanometers in size $[27,28]$. PS has a number of useful properties. Owing to wider bandgap, intense visible photoluminescence, and a large surface area per unit volume, PS nanostructures are promising for photoelectronics, touch-sensor electronics and biomedical technologies [29-32]. Therefore, significant efforts have been devoted to the growth of $\mathrm{ZnO}$ nanostructures on the PS substrates [16,21,26,33]. ZnO-PS nanocomposites have been used for white light emission, photodetectors and to tune $\mathrm{ZnO}$ grain size for possible sensing applications. Less attention so far has been paid to the studies of charge transport in $\mathrm{ZnO}-\mathrm{PS}$ nanosystems. Understanding the conductivity mechanisms, however, is important from a perspective of LED or photoelectric conversion applications.

In the present research, $\mathrm{ZnO}$ nanostructures were grown by means of electrodeposition method on the PS-silicon 
substrate. Specific attention was focused on exploring the influence of $\mathrm{ZnO}$ layers on the electrical properties of PS nanostructures. Studies of the relaxation processes in the structures based on PS were performed using thermally stimulated depolarization (TSD) spectroscopy.

\section{Experiment}

In the experimental studies we used PS samples that were fabricated by means of electrochemical etching of single-crystalline (100) silicon substrates with a thickness of $400 \mu \mathrm{m}$ in ethanol solution of hydrofluoric acid. The silicon substrates have the $n$-type conductivity with the specific resistance of $4.5 \mathrm{Ohm} \cdot \mathrm{cm}$. In order to obtain homogeneous layers, gold films were preliminary deposited on back surfaces of the substrates with the aid of a thermo-vacuum technique. These films also served as contacts in further measurements. Anodic current density was set to $30 \mathrm{~mA} / \mathrm{cm}^{2}$ throughout the etching period. The duration of etching process was $10 \mathrm{~min}$. To ensure the availability of holes in the surface layer of $n-\mathrm{Si}$, which is necessary for anodic reactions to occur and allows the formation of PS [27], the working surface of a silicon plate was irradiated with white light during the entire process of electrochemical etching.

In order to obtain $\mathrm{ZnO}$ layers on the PS surface, electrochemical deposition method was used. The method has proven itself effective in the growth of $\mathrm{ZnO}$ nanostructures on $\mathrm{Si}$ and other electrically conductive substrates $[5,8]$. Two-electrode electrochemical cell with water solution of $0.05 \mathrm{M} \mathrm{Zn}\left(\mathrm{NO}_{3}\right)_{2} \cdot 6 \mathrm{H}_{2} \mathrm{O}$ and $0.1 \mathrm{M} \mathrm{NaNO}_{3}$ was used in the deposition process. The deposition potential of $-1.4 \mathrm{~V}$ was applied to the porous silicon-silicon substrate, which served as a working electrode. The platinum net played a role of the additional electrode. Duration of growth for $\mathrm{ZnO}$ nanostructures was $10-20 \mathrm{~min}$. Electrolyte was heated up to $65-80{ }^{\circ} \mathrm{C}$ in the deposition process, since the temperature is one of the defining factors influencing $\mathrm{ZnO}$ morphology. During the electrochemical deposition, the reagents penetrate into the pores of silicon and zinc oxide is synthesized right on the surface of the electrode and inside the pores. After the process of electrodeposition, the experimental samples were washed in distilled water and dried in air. For the purpose of experimental studies, $\mathrm{ZnO}$ PS-Si structures obtained by deposition of zinc oxide onto PS at $\tau=10 \mathrm{~min}, t=65^{\circ} \mathrm{C}$ (sample A), $\tau=20 \mathrm{~min}, t=75^{\circ} \mathrm{C}$ (sample B) and $\tau=20 \mathrm{~min}, t=80^{\circ} \mathrm{C}$ (sample C) were used.

$\mathrm{ZnO}-\mathrm{PS}-\mathrm{Si}$ hybrid structures were characterized by scanning electron microscopy (SEM). After SEM investigation, the contact with a diameter of $\sim 3 \mathrm{~mm}$ was deposited on the surface of PS and $\mathrm{ZnO}$ layer using conductive paste. Hence, the investigations of electrical properties of experimental samples were carried out in the geometry in which the current flows across the structure and perpendicular to the surface.

Digital RLC-meter working at $1 \mathrm{MHz}$ frequency was employed to study temperature dependencies of electric conductivity of PS-based structures. Samples under study were placed into the cryostat kept under $10^{-3} \mathrm{mmhg}$ vacuum. In the TSD experiment, experimental structures were polarized (polarization voltage was $U=5 \mathrm{~V}$ ) at room temperature and then cooled down to liquid nitrogen temperature. Investigation of the temperature dependence of the depolarization current of preliminary polarized samples was carried out in the absence of the external electric field at linear heating from 80 to $325 \mathrm{~K}$. The heating rate was 0.1 $\mathrm{K} / \mathrm{sec}$.

\section{Result and Discussion}

Analysis of the surface and the cross section of $\mathrm{ZnO}-\mathrm{PS}-$ $\mathrm{Si}$ structures were carried out using SEM methods in elastically reflected electrons mode and X-ray microanalysis (Figure 1).

As one can see from Figure 1,a, the formation of narrow pores was observed. Pores were oriented perpendicular to the silicon surface. According to SEM studies of the porous layer, its thickness was about $20 \mu \mathrm{m}$. Average pore diameter is within the range of 100-1000 nm. Dense arrays of $\mathrm{ZnO}$ nanostructures formed a layer on the surface of PS and partially penetrated into the pores. Surface morphology of $\mathrm{ZnO}$ arrays depended on the temperature of the electrolyte and electrodeposition modes.

At $65{ }^{\circ} \mathrm{C}$, the formation of $\mathrm{ZnO}$ nanowires network was observed. At elevated temperatures and prolonged electrodeposition durations, disordered arrays of zinc oxide nanocrystals were formed. At $80{ }^{\circ} \mathrm{C}$, disordered nanorods of $\mathrm{ZnO}$ were formed; the smaller rods were grown on larger hexagonal ones, with sizes up to several microns (see Figure 1,d). The broad spectrum of $\mathrm{ZnO}$ morphological peculiarities is due to different kinetic parameters of different growth planes [3]. The important factor that allows to effectively control the shape and the size of $\mathrm{ZnO}$ arrays is the temperature of the electrolyte, since it determines the intensity of electrochemical processes, reagent diffusion rate and desorption rate of reaction products from the surface of grown $\mathrm{ZnO}$ [34]. The thickness of zinc oxide layer was increased with deposition duration. The X-ray surface microanalysis of the hybrid structures revealed the traces of silicon, zinc and oxygen. This fact proves that the surface of PS was covered by $\mathrm{ZnO}$ layer. 

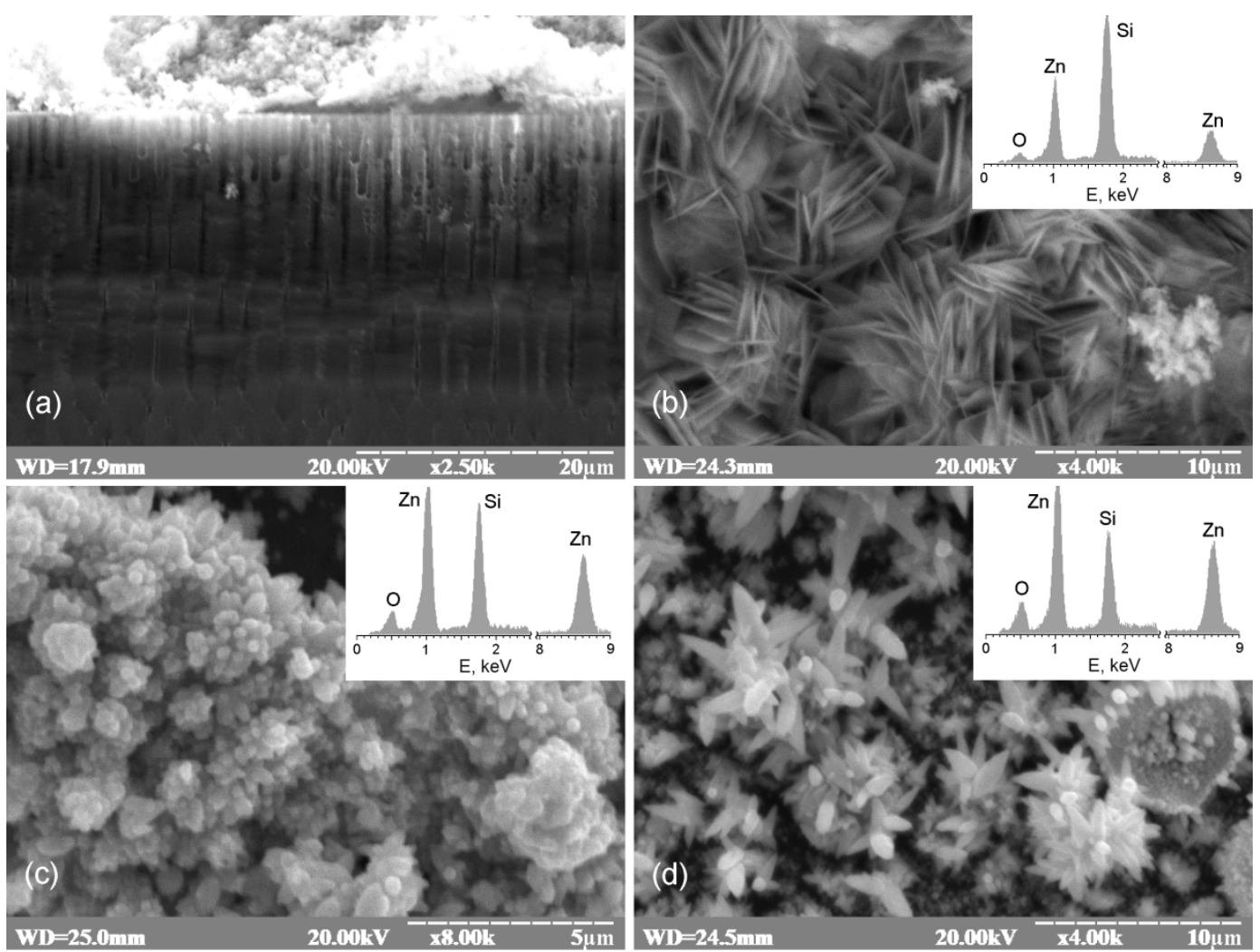

Figure 1. SEM images of the ZnO-PS-Si cross section (a) and the surface of arrays of $\mathrm{ZnO}$ nanostructures, deposited on PS at $\tau=10 \mathrm{~min}, t=65^{\circ} \mathrm{C}(\mathrm{b}), \tau$ $=20 \mathrm{~min}, t=75^{\circ} \mathrm{C}(\mathrm{c}), \tau=20 \mathrm{~min}, t=80^{\circ} \mathrm{C}$ (d). Insets: X-ray surface microanalysis of the ZnO-PS-Si structures

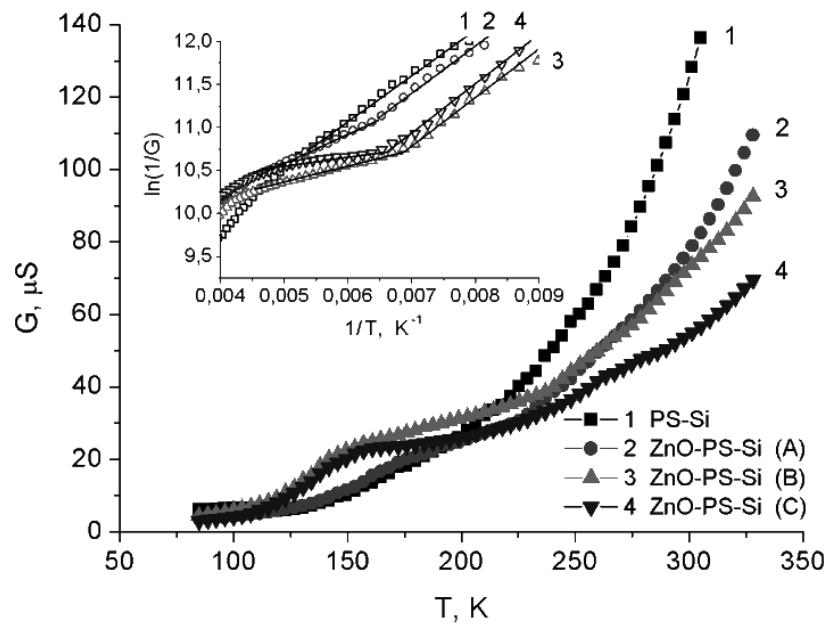

Figure 2. Temperature dependencies of electric conductivity for PS-Si (1) and $\mathrm{ZnO}-\mathrm{PS}-\mathrm{Si}(2,3,4)$ structures. Inset shows the same temperature dependence in $\left(\ln G^{-1}, T^{-1}\right)$ coordinates

In order to clarify the mechanism of charge transport in PS-Si and ZnO-PS-Si structures, temperature dependencies of electric conductivity $G$ in the range of $80-325 \mathrm{~K}$ were studied. Exponential increase in the conductivity of experimental samples at elevating temperatures was detected, as shown in Figure 2. This indicates the activation mechanism of charge transport in investigated structures [35]. Based on the temperature dependence of the resistivity, presented in $(\ln 1 / G, 1 / T)$ coordinates, one can estimate the activation energy for the conductivity (see inset in Figure 2). The activation energy for PS-Si structures in the low temperature range, as calculated by measuring the slope of $\ln$ $G^{-1}\left(T^{-1}\right)$ line was about $0.05 \mathrm{eV}$.

$G(T)$ dependence for $\mathrm{ZnO}-\mathrm{PS}-\mathrm{Si}$ structures exhibits two characteristic regions: around 80-140 K and around 140-250 $\mathrm{K}$, where activation energy for conductivity is different. In the low-temperature region the activation energy for conductivity in case of ZnO-PS-Si structures is $0.05 \mathrm{eV}$ which is close to that of PS-Si. In 140-250 K region, the activation energy of charge transport in $\mathrm{ZnO}-\mathrm{PS}-\mathrm{Si}$ structures is dependent on the morphology of $\mathrm{ZnO}$ arrays and is found to be $0.03,0.02$ and $0.013 \mathrm{eV}$ for samples A, B and $\mathrm{C}$, respectively. This might be related to disordered character of $\mathrm{ZnO}-\mathrm{PS}$ nanocomposite structure. Generally, disordered semiconductors not only have different conductivity activation energy values, but also allow for several simultaneous charge transport mechanism to be realized. The role of such mechanisms depends on the temperature range [36]. Besides, closer to ambient temperature we observed lower values of $\mathrm{ZnO}-\mathrm{PS}-\mathrm{Si}$ conductivity in comparison with PS-Si, what may due to larger electrical resistance of wide band gap zinc oxide nanocrystals. Moreover, electrical conductivity of the samples A, B and C decreased with the increase of the size of $\mathrm{ZnO}$ structures (see Figure 2).

Studies of the relaxation processes in the structures based on PS were performed using TSD spectroscopy. In the case of disordered systems, including PS, the trap levels are distributed quasi-continuously on the activation energy. To 
determine the activation energy of electrically active defects in experimental structures, the temperature dependencies of the depolarization current have been measured.

TSD spectra of PS-Si and $\mathrm{ZnO}-\mathrm{PS}-\mathrm{Si}$ show similar character. There occurs thermally stimulated (from LNT temperatures) emission of charge carriers, then one observes an increase in current with temperature and the band with the maximum current in 250-270 K range (Figure 3).

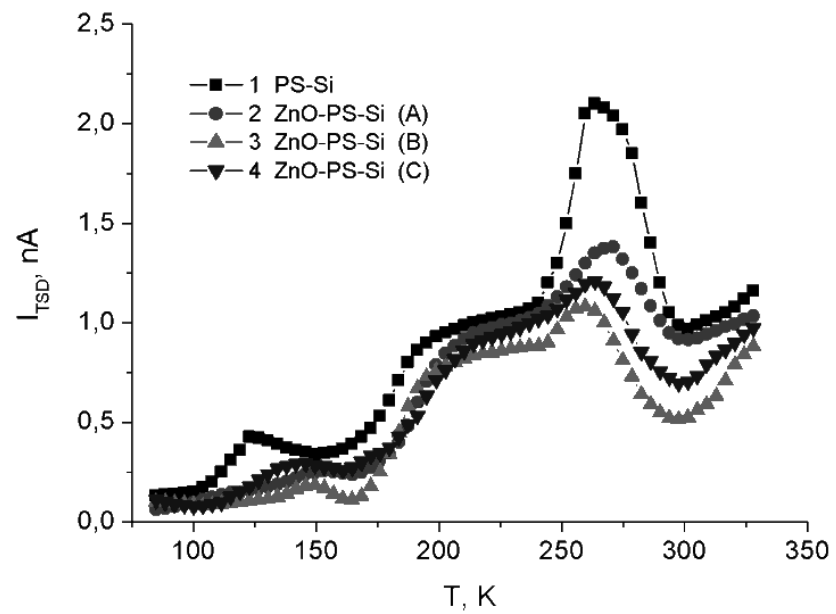

Figure 3. Temperature dependencies of depolarization current for PS-Si (1) and $\mathrm{ZnO}-\mathrm{PS}-\mathrm{Si}(2,3,4)$ structures

In the TSD spectra of $\mathrm{ZnO}-\mathrm{PS}-\mathrm{Si}$ structures the values of depolarization current in 120-130 $\mathrm{K}$ range are lower and the maximum current band is shifted towards higher temperatures in comparison with the depolarization spectrum of PS-Si. The TSD spectra of different $\mathrm{ZnO}-\mathrm{PS}-$ Si structures were very similar. This indicates the similar activation energy distribution of electrically active defects in experimental structures.

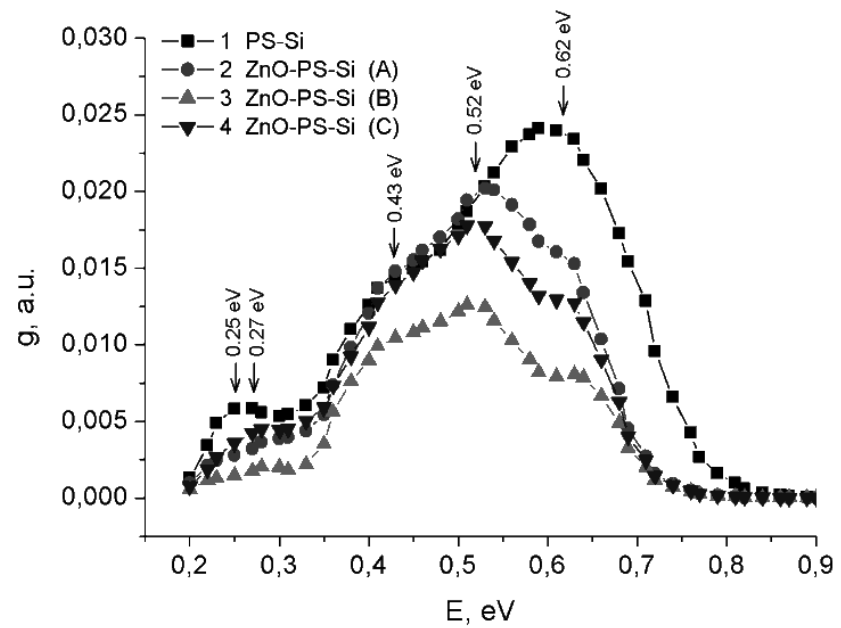

Figure 4. Energy distribution of the density of states in PS-Si (1) and $\mathrm{ZnO}-\mathrm{PS}-\mathrm{Si}(2,3,4)$ structures

The analysis of the TSD spectra was performed based on the phenomenological theory of TSD currents for the disordered dielectrics [37]. The results of the calculation of the density of states energy distribution $g(E)$, which was carried out by the numerical method based on the Tikhonov regularization algorithm, are presented in Figure 4. Approximation of the energy distribution with Gaussian functions suggests that the groups of trap levels, with differences in nature and activation energies in the ranges of $0.2-0.3,0.4-0.45$ and $0.6-0.65 \mathrm{eV}$ are present.

Drawing the correlations with $\mathrm{SiO}_{2}$, one can suggest that the $0.2-0.3 \mathrm{eV}$ band corresponds to the activation energy of hydrogen ions $\mathrm{H}^{+}$[38]. It is likely that the bands at $0.4-0.45$ and $0.6-0.65 \mathrm{eV}$ are associated with the electrically active defects in the PS layer. In the density of states energy spectra for $\mathrm{ZnO}-\mathrm{PS}-\mathrm{Si}$ structures one can emphasize the additional band at $0.5-0.55 \mathrm{eV}$, which is probably related to the defects at $\mathrm{Si}-\mathrm{ZnO}$ interface, because this band is observed for samples with different $\mathrm{ZnO}$ morphology. The layer of $\mathrm{ZnO}$ nanocrystals at PS surface is responsible also for the transformation of $0.2-0.3 \mathrm{eV}$ band.

\section{Conclusions}

It has been shown that electrochemical deposition is an efficient method for controlled growth of the arrays of zinc oxide nanostructures on PS surface. The shape and size of such arrays can be largely tuned by changing temperature and temporal parameters of electric deposition. In turn, morphological features of the zinc oxide layers determine the electric conductivity of $\mathrm{ZnO}-\mathrm{PS}-\mathrm{Si}$ structures. Decrease in the conductivity of such structures as compared to the initial PS-Si sample at room temperature may be related to larger electrical resistance of wide band gap $\mathrm{ZnO}$ nanocrystals.

By performing complex studies of electrical conductivity and depolarization current in the wide temperature range, charge transport and relaxation processes in PS-based structures were analyzed. Activation mechanism of electrical conductivity was established and the respective activation energy for different temperature ranges was estimated. For PS-Si structure this energy is $0.05 \mathrm{eV}$ in the $80-240 \mathrm{~K}$ temperature range. $\mathrm{ZnO}$ layers lead to the decrease of the electric conductivity activation energy in 140-250 K temperature range. Activation energy of charge transport in $\mathrm{ZnO}-\mathrm{PS}-\mathrm{Si}$ structures is within $0.013-0.03 \mathrm{eV}$ and depends on the morphology of $\mathrm{ZnO}$ layers.

As follows from the analysis of TSD spectra of PS-Si structure, the energy distribution of the density of states for nonequilibrium carriers $g(E)$ shows peaks in the ranges of $0.2-0.3,0.4-0.45$ and $0.6-0.65 \mathrm{eV}$. $\mathrm{ZnO}$ nanocrystals grown on porous silicon substrates modify the density of states in different energy ranges and lead to the appearance of the additional band at $0.5-0.55 \mathrm{eV}$. Such localized electron states influence the charge transport in the ZnO-PS nanostructures.

\section{REFERENCES}

[1] S. Mridha, D. Basak. Effect of thickness on the structural, 
electrical and optical properties of $\mathrm{ZnO}$ films, Materials Research Bulletin, Vol.42, No.5, 875-882, 2007.

[2] Y. W. Heo, D. P. Norton, L. C. Tien, Y. Kwon, B. S. Kang, F. Ren, S. J. Pearton, J. R. LaRoche. ZnO nanowire growth and devices, Materials Science and Engineering: R: Reports, Vol.47, No.1, 1-47, 2004.

[3] Z. L. Wang. Zinc oxide nanostructures: growth, properties and applications, Journal of Physics: Condensed Matter, Vol. 16, No. 25, R829-R858, 2004.

[4] V. A. Fonoberow, A. A. Khan, A. A. Balandin, F. Xiu, J. Liu. Photoluminescence investigation of the carrier recombination processes in $\mathrm{ZnO}$ quantum dots and nanocrystals, Physical Review B, Vol.73, 165317, 2006.

[5] V. Kapustianyk, B. Turko, I. Luzinov, V. Rudyk, V. Tsybulskyi, S. Malynych, Yu. Rudyk, M. Savchak. LEDs based on p-type $\mathrm{ZnO}$ nanowires synthesized by electrochemical deposition method, Physica Status Solidi (C), Vol.11, No.9-10, 1501-1504, 2014.

[6] I. C. Yao, T. Y. Tseng, P. Lin. ZnO nanorods grown on polymer substrates as UV photodetectors, Sensors and Actuators A: Physical, Vol.178, 26-31, 2012.

[7] H. Kind, H. Yan, B. Messer, M. Law, P. Yang. Nanowire ultraviolet photodetectors and optical switches, Advanced Materials, Vol.14, No.2, 158-160, 2002.

[8] N. P. Klochko, K. S. Klepikova, G. S. Khrypunov, N. D. Volkova, V. R. Kopach, V. M. Lyubov, M. V. Kirichenko, A. V. Kopach. Antireflective nanostructured zinc oxide arrays produced by pulsed electrodeposition, Semiconductors, Vol.49, No.2, 214-223, 2015.

[9] Z. R. Tian, J. A. Voigt, J. Liu, B. Mckenzie, M. J. McDermott, M. A. Rodriguez, H. Konishi, H. Xu. Complex and oriented ZnO nanostructures, Nature Materials, Vol.2, 821-826, 2003.

[10] I. C. Yao, P. Lin, T. Y. Tseng. Hydrogen gas sensors using $\mathrm{ZnO}-\mathrm{SnO}_{2}$ core-shell nanostructure, Advanced Science Letters, Vol.3, 548-553, 2010.

[11] J. J. Delaunay, N. Kakoiyama, I. Yamada. Fabrication of three-dimensional network of $\mathrm{ZnO}$ tetratpods and its response to ethanol, Materials Chemistry and Physics, Vol.104, No.1, 141-145, 2007.

[12] S. C. Ko, Y. C. Kim, S. S. Lee, S. H. Choi, S. R. Kim. Micromachined piezoelectric membrane acoustic device, Sensors and Actuators A: Physical, Vol.103, 130-134, 2003.

[13] M. W. Zhu, N. Huang, J. Gong, B. Zhang, Z. J. Wang, C. Sun, $\mathrm{X}$. Jiang. Growth of $\mathrm{ZnO}$ nanorod arrays by sol-gel method: transition from two-dimensional film to one-dimensional nanostructure, Applied Physics A, Vol.103, 159-166, 2011.

[14] J. J. Wu, S. C. Liu. Low-temperature growth of well-aligned $\mathrm{ZnO}$ nanorods by chemical vapor deposition, Advanced Materials, Vol.14, 215-218, 2002.

[15] K. M. K. Srivatsa, D. Chhikara, M. S. Kumar. Synthesis of aligned $\mathrm{ZnO}$ nanorod array on silicon and sapphire substrates by thermal evaporation technique. Journal of Materials Science \& Technology, Vol.27, No.8, 701-706, 2011.

[16] H. I. Abdulgafour, F. K. Yam, Z. Hassan, K. Al-Heuseen, M. J. Jawad. $\mathrm{ZnO}$ nanocoral reef grown on porous silicon substrates without catalyst, Journal of Alloys and Compounds,
Vol.509, 5627-5630, 2011.

[17] K. B. Sundaram, A. Khan. Characterization and optimization of zinc oxide films by r.f. magnetron sputtering, Thin Solid Films, Vol.295, No.1, 87-91, 1997.

[18] J. H. Yang, J. H. Zheng, H. J. Zhai, L. L. Yang. Low temperature hydrothermal growth and optical properties of $\mathrm{ZnO}$ nanorods. Crystal Research and Technology, Vol.44, 87-91, 2009.

[19] J. N. Zeng, J. K. Low, Z. M. Ren, T. Liew, Y. F. Lu. Effect of deposition conditions on optical and electrical properties of $\mathrm{ZnO}$ films prepared by pulsed laser deposition, Applied Surface Science, Vol.197-198, 362-367, 2002.

[20] V. V. Gafiychuk, B. K. Ostafiychuk, D. I. Popovych, I. D. Popovych, A. S. Serednytski. ZnO nanoparticles produced by reactive laser ablation, Applied Surface Science, Vol.257, No.20, 8396-8401, 2011.

[21] L. Martinez, O. Ocampo, Y. Kumar, V. Agarwal. ZnO-porous silicon nanocomposite for possible memristive device fabrication, Nanoscale Research Letters, Vol.9, 437, 2014.

[22] M. Bechelany, A. Amin, A. Brioude, D. Cornu, P. Miele. ZnO nanotubes by template-assisted sol-gel route, Journal of Nanoparticle Research, Vol.14, 980, 2012.

[23] V. Manthina, T. Patel, A. G. Agrios. Number density and diameter control of chemical bath deposition of $\mathrm{ZnO}$ nanorods on FTO by forced hydrolysis of seed crystals, Journal of the American Ceramic Society, Vol.97, No.4, 1028-1034, 2014.

[24] D. Gedamu, I. Paulowicz, S. Kaps, O. Lupan, S. Wille, G. Haidarschin, Y. K. Mishra, R. Adelung. Rapid fabrication technique for interpenetrated $\mathrm{ZnO}$ nanotetrapod networks for fast UV sensors, Advanced Materials, Vol. 26, 1541-1550, 2014.

[25] V. A. Antohe, L. Gence, S. K. Srivastava, L. Piraux. Template-free electrodeposition of highly oriented and aspect-ratio controlled $\mathrm{ZnO}$ hexagonal columnar arrays. Nanotechnology, Vol.23, No.25, 255602, 2012.

[26] H. C. Hsu, C. S. Cheng, C. C. Chang, S. Yang, C. S. Chang, W. F. Hsieh. Orientation-enhanced growth and optical properties of $\mathrm{ZnO}$ nanowires grown on porous silicon substrates. Nanotechnology, Vol.16, No.2, 297-301, 2005.

[27] A. G. Cullis, L. T. Canham, P. D. J. Calcott. The structural and luminescence properties of porous silicon, Journal of Applied Physics, Vol.82, 909-965, 1997.

[28] H. Föll, M. Christophersen, J. Carstensen, G. Hasse. Formation and application of porous silicon, Materials Science and Engineering: R: Reports, Vol.39, No.4, 93-141, 2002.

[29] B. Ünal, A. N. Parbukov, S. C. Bayliss. Photovoltaic properties of a novel stain etched porous silicon and its application in photosensitive devices, Optical Materials, Vol.17, 79-82, 2001.

[30] I. B. Olenych, O. I. Aksimentyeva, L. S. Monastyrskii, Y. Y. Horbenko, L. I. Yarytska. Sensory properties of hybrid composites based on poly (3,4-ethylenedioxythiophene)porous silicon-carbon nanotubes, Nanoscale Research Letters, Vol.10, 187, 2015. 
[31] I. B. Olenych, L. S. Monastyrskii, O. I. Aksimentyeva, B. S. Sokolovskii. Effect of bromine adsorption on the charge transport in porous silicon - silicon structures, Electronic Materials Letters, Vol.9, No.3, 257-260, 2013.

[32] I. Rea, A. Lamberti, I. Rendina, G. Coppola, M. Gioffrè, M. Iodice, M. Casalino, E. De Tommasi, L. De Stefano, Fabrication and characterization of a porous silicon based microarray for label-free optical monitoring of biomolecular interactions, Journal of Applied Physics, Vol.107, 014513, 2010.

[33] R. G. Singh, F. Singh, V. Agarwal, R. M. Mehra. Photoluminescence studies of $\mathrm{ZnO}$ /porous silicon nanocomposites, Journal of Physics D: Applied Physics, Vol. 40, 3090-3093, 2007.

[34] N. P. Klochko, Y. O. Myagchenko, E. E. Melnychuk, V. R. Kopach, E. S. Klepikova, V. N. Lyubov, G. S. Khrypunov, A.
V. Kopach. Prospects for the pulsed electrodeposition of zinc-oxide hierarchical nanostructures, Semiconductors, Vol.47, No.8, 1123-1129, 2013.

[35] L. M. Sorokin, V. I. Sokolov, A. E. Kalmykov, A. V. Chernyaev. Features of the current-voltage characteristics and temperature dependences of electric conductivity in porous silicon layers, Technical Physics Letter, Vol.36, 1146-1149, 2010.

[36] N. F. Mott, E. A. Davis, Electronic Processes in Non-Crystalline Materials, Clarendon Press, Oxford, 1979.

[37] Y. Gorohovatsky, H. Bordovsky, Thermally Activational Current Spectroscopy of Highresistance Semiconductors and Dielectrics, Nauka, Moscow, 1991.

[38] L. S. Monastyrskii, T. I. Lesiv, I. B. Olenych. Composition and properties of thin films on porous silicon surface, Thin Solid Films, Vol.343-344, 335-337, 1999. 